\title{
Vielfalt als Erfolgsfaktor
}

\section{Diversity Management als Aufgabe auch in sozialen Diensten und Einrichtungen}

\section{SUSANNE WITTMANN}

Susanne Wittmann studierte an der Evangelischen Fachhochschule Rheinland-Westfalen-Lippe in Bochum Soziale Arbeit und Management in sozialwirtschaftlichen und diakonischen Organisationen. Sie ist seit zwölf Jahren bei dem Verein für multikulturelle Kinder- und Jugendhilfe - Migrationsarbeit in Bochum (IFAK e. V.) im Bereich Migration und Erwachsenenbildung sowie Projektarbeit tätig.

www.ifak-bochum.de

\author{
Diversity Management bezeichnet das konsequente \\ Bemühen, in einer Organisation die Vielfalt in der \\ Mitarbeiterschaft hinsichtlich Geschlecht, Herkommen, \\ Alter, Behinderung, Religion und Lebensstil zu \\ fördern. Dabei steht nicht die Minderheit selbst im \\ Fokus, sondern die Gesamtheit der Mitarbeitenden \\ in ihren Unterschieden und Gemeinsamkeiten.
}

Diversity Management hat seine Wurzeln in den USA, wo sich die Bürgerrechtsbewegung in den 1960er Jahren für den Schutz gegen Diskriminierung aufgrund von Rasse, Hautfarbe, Religion, Geschlecht und nationaler Herkunft einsetzte (vgl. Vedder 2006).

Nach heutigem Verständnis zielt Diversity Management darauf ab, die Vielfalt in der Zusammensetzung der Mitarbeitenden in Unternehmen hinsichtlich Alter, Geschlecht, ethnischer Herkunft, physischer Fähigkeiten, sexueller Orientierung, Weltanschauung usw. als Vorteil für den Unternehmenserfolg wertzuschätzen und $\mathrm{zu}$ fördern. International operierende Unternehmen haben die Bedeutung des Ansatzes der kulturellen Diversifikation schon sehr früh erkannt und genutzt. Durch den Wandel des Arbeitsmarktes gewinnt Diversity Management in seinen verschiedenen Facetten auch in sozialen Organisationen zunehmend an Bedeutung.

Diversity Management (synonym: Managing Diversity) gilt in Unternehmen als Strategie, um auf den verändernden Personalmarkt (workforce diversity) einzuwirken und die eigene Organisation diversitätsgerecht (workplace diversity) zu gestalten (vgl. Krell u.a. 2007, 9). Entsprechend ist das Konzept eng mit Organisationsentwicklung verbunden. Aufbauend auf der Diagno- se, dass qualifizierte personale Ressourcen knapp sind, sind organisatorische Strukturen zu verändern, um unterschiedliche personale Potenziale gezielt erkennen, entwickeln und fördern zu können.

Der Begriff »Diversity“, als Vielfalt, Heterogenität, Verschiedenheit, wird zurzeit eng mit dem ökonomischen Konzept gepaart (vgl. Perko/Czollek 2008), das die Bindung von Mitarbeitenden an Unternehmen, die Akquisition von neuen Zielgruppen und die Erweiterung bestehender Kundenbeziehungen verfolgt. Diese Entwicklung hat zu dem Urteil geführt, Diversity Management diene in erster Linie profitorientierten $\mathrm{Zie}$ len, während die ursprünglichen Ziele aus den 1960er Jahren, hauptsächlich Antidiskriminierung, nur noch eine untergeordnete Rolle spielten (vgl. Krell/ Sieben 2010, 45).

Dieses Urteil ist nicht ganz von der Hand zu weisen. Daran ändert sich auch nichts, wenn Unternehmen im Kontext von Corporate Social Responsibility (CSR) ihr Handeln ethisch-moralisch begründen. Ein Element solch einer Begründung ist der Bezug auf Diversity Management, das Verfolgen gleichstellungspolitischer Ziele. Für sozialwirtschaftliche Organisationen ist die »Equity Perspektive (vgl. Vedder 2006) nicht nur aus ökonomischen Gründen beachtenswert. Der diskrimi- 
nierungsfreie Umgang und Bezug auf Menschen sollte für diese Organisationen Ausdruck ihres gelebten Selbstverständnisses sein.

Ausgehend von einer integrativen Position zielt Diversity Management darauf, Nachteile zu reduzieren und Chancen zu realisieren zum Nutzen von Organisationen und deren Mitarbeitenden. Diese Position bewegt sich aufgrund unterschiedlicher Interessen notwendig in einem Spannungsfeld. Der Umgang mit möglichen Konflikten stellt die Organisationen und ihre Führungskräfte vor Herausforderung, deren Bewältigung Gegenstand von Managementhandeln ist (vgl. Koall $\mathrm{u}$. a. 2007).

$\mathrm{Zu}$ den betriebswirtschaftlichen Zielen des Managing Diversity zählen Effizienzsteigerung durch höhere Motivation und weniger Diskriminierung, Verbesserung der organisatorischen Flexibilität durch vielfältige Teams, Erhöhung von Kreativität und Innovationsfähigkeit bei Problemlösungen, größere Auswahl an Bewerbern und Bewerberinnen, stärkere Kundenorien-
Praktiken eingefordert. Zudem werden in dem Kontext Diskriminierungen jedweder Art thematisiert. Darauf aufbauend werden alternative und partizipative Konzepte entwickelt.

\section{Vielfalt meint Unterschiede und Gemeinsamkeiten}

Vielfalt bezieht sich sowohl auf gruppenbildende Kategorien, wie Geschlecht, Sprache, Herkunft, als auch auf individuelle Aspekte wie Werte, Einstellungen und Verhalten. In der Literatur finden sich zum Begriff Vielfalt zwei Verständnisse, zum einen »Vielfalt als Unterschiede « (vgl. Loden/Rosener 1991, 18) und zum anderen "Vielfalt als Unterschiede und Gemeinsamkeiten« (vgl. Thomas 1996, 5).

Wird Vielfalt auch als Gemeinsamkeit verstanden, so spiegelt sich darin die Tatsache, dass Menschen immer auch mehreren Gruppen angehören (vgl. Krell 2010, 50), dass es innerhalb der Gruppen Unterschiede gibt und zwischen ihnen Gemeinsamkeiten. Krell weist darauf hin, dass das Ver-

"Menschen mit nichtdeutschen Namen haben signifikant weniger Chancen,

\section{zu einem Bewerbungsgespräch}

\section{eingeladen zu werden«}

tierung, verbesserter Umgang mit der zunehmenden Internationalisierung und Aufwertung des Images von Unternehmen und Organisationen.

Neben der ökonomischen lassen sich weitere Perspektiven für Diversity Management anführen. Unter rechtlichem Gesichtspunkt wird auf eine Reihe von Gesetzen, wie das Grundgesetz, das Betriebsverfassungsgesetz und das Allgemeine Gleichbehandlungsgesetz verwiesen. Wird die »Equity Perspektive « in den Vordergrund gestellt und ethisch-moralisch argumentiert, dann werden zwangsläufig monokulturelle Organisationen, geprägt durch eine dominante Gruppe, infrage gestellt. Aus dieser Perspektive werden vorurteils- und diskriminierungsfreie personalpolitische Kriterien, Verfahren und ständnis »Vielfalt als Unterschiede und Gemeinsamkeiten« weniger anfällig für Festschreibungen ist.

Ein Verständnis von Diversity, das nur auf Unterschiede fokussiert, birgt die Gefahr der Stereotypisierung. Das ist nicht nur problematisch, wenn Personen aufgrund bestimmter Zugehörigkeiten bestimmte Defizite zugeschrieben werden, sondern auch, wenn es um die Zuschreibung besonderer Potenziale geht. Dies führt mitunter zu strukturellen Bevorzugungen oder Benachteiligungen, die sich in unterschiedlichen Zugangs- und Teilhabechancen auf dem Arbeitsmarkt spiegeln. Diskriminierung auf dem Arbeitsmarkt kann als Prozess definiert werden, bei dem Mitglieder einer bestimmten demografischen Gruppe unterschiedliche Behandlung erfahren, die nicht auf ihre Produktivität zurückzuführen ist.

Dass bei der Implementierung von Diversity Management das Bemühen um Antidiskriminierung nicht (notwendigerweise) das Antriebsrad bildet, wird deutlich, wenn sich Organisationen nur so divers geben, wie es ökonomisch erfolgversprechend und rentabel erscheint. Der solch einem Verhalten zugrundeliegende Nützlichkeits-Rassismus transformiert Diversität zu einem Instrument, dessen Einsatz wiederum selektiv und damit diskriminierend ist. So verhält es sich beispielsweise mit der Unterzeichnung der Kommunen zur Charta der Vielfalt und der Entwicklung von Städteleitbildern. Diese Selbstverpflichtung wird in vielen Städten von Experten als strategischer Wettbewerbsvorteil angepriesen.

\section{Ein praktisches Beispiel: anonyme Bewerbungen}

Unter der Anwendung von Maßnahmen des Diversity Managements sollen Organisationen sich so verändern, dass sie für Menschen aller Gruppenzugehörigkeiten und Individualitäten die gleichen Zugangs- und Teilhabechancen ermöglichen (vgl. Krell/Sieben 2010, 47). Dem Wandel von einer monokulturellen zu einer multikulturellen Organisation dienen verschiedene Schritte. Hierzu zählen u. a. ein Diversity-Audit, die Verankerung von Diversity Management in der Organisationskultur, DiversityTrainings, sowie Verfahren und Praktiken der Personalpolitik. Ein Beispiel innerhalb der Personalauswahl für eine diskriminierungsfreie Methode ist das anonyme Bewerbungsverfahren.

Dies führt zwar nicht zwingend zu Personalvielfalt, jedoch soll dadurch sichergestellt werden, dass ausschließlich qualifikatorische Gesichtspunkte bei der Personalauswahl eine Rolle spielen. Klassische Beurteilungsfehler, wie beispielsweise der Stereotypen-Effekt können mit diesem Instrument vermieden werden (vgl. Stock-Homburg 2010, $384 \mathrm{ff})$. So zeigt eine Studie des Instituts Zukunft der Arbeit (IZA), dass die Chance, zum Bewerbungsgespräch eingeladen zu werden, bei Bewerbern und Bewerberinnen mit nichtdeutschen $\mathrm{Na}$ men um 14 Prozent sinkt und bei kleineren Betrieben sogar um 24 Prozent (vgl. Krause/Rinne/Zimmermann 2010). $\Rightarrow$ 


\section{Wie funktionieren eigentlich Bewerbungsverfahren ohne persönliche Angaben?}

Wichtig ist, dass die Einladung zum Vorstellungsgespräch ausschließlich aufgrund der Qualifikation erfolgt. Bei anonymisierten Bewerbungen wird daher zunächst auf ein Foto der sich bewerbenden Person, ihren Namen, die Adresse, das Geburtsdatum oder Angaben zu Alter, Familienstand oder Herkunft verzichtet. Abgesehen davon können alle üblichen Informationen abgefragt werden, wie etwa Berufserfahrung, Ausbildung, Motivation usw. An dieser Stelle gibt es keinen signifikanten Unterschied zu herkömmlichen Lebensläufen - außer dem Verzicht auf Jahreszahlen. In der ersten Auswahlrunde wird der Blick der Personalerinnen und Personaler ausschließlich auf die Qualifikation der Bewerbenden gelenkt. In der zweiten Phase, wenn die Einladung zum Vorstellungsgespräch ausgesprochen ist, erhalten Personalerinnen und Personaler vollständige Unterlagen mit persönlichen Angaben und können sich auf das Gespräch vorbereiten. Sie sitzen also nicht - wie oft angenommen wird - vor einer ihnen völlig unbekannten Person.

Die Antidiskriminierungsstelle des Bundes ist eine unabhängige Anlaufstelle für Menschen, die von Diskriminierung betroffen sind. Sie wurde im jahre 2006 eingerichtet, nachdem das Allgemeine Gleichbehandlungsgesetz (AGG) in Kraft getreten ist. Ein Projekt der Antdiskriminierungsstelle des Bundes ist der Versuch, anonyme Bewerbungen in deutschen Unternehmen einzuführen. Dazu gibt es eine eigene Website mit Hinweisen für Bewerber und Arbeitgeber.

www.antidiskriminierungsstelle.de/DE/Projekte_ADS/anonymisierte_bewerbungen/ anonymisierte_bewerbungen_node.html

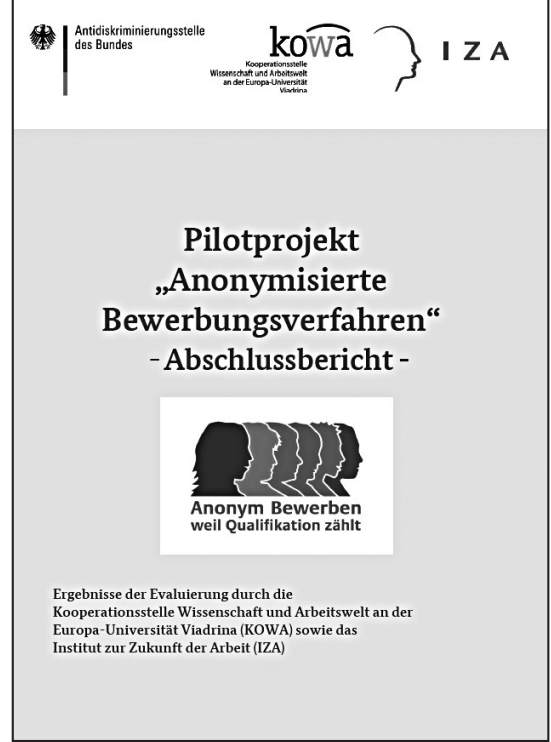

Die Antidiskriminierungsstelle des Bundes startete im Herbst 2010 das Pilotprojekt »Anonyme Bewerbung«. Fünf Konzerne werten ein Jahr lang nur noch anonymisierte Bewerbungen aus, ebenfalls das Bundesfamilienministerium und das Integrationsministerium Nordrhein-Westfalen. Auch die Landesverwaltung in Nordrhein-Westfalen startete im Januar 2011 mit der Initiative »Anonyme Bewerbung «. Es bleibt abzuwarten, ob und wann Organisationen der Sozialwirtschaft diesem Beispiel folgen.

\section{Literatur}

Koall, I./Bruchhagen, V./Höher, F. (Hg.): Diversity Outlooks. Managing Diversity zwischen Ethik, Profit und Antidiskriminierung. Hamburg 2007.

Krause, A./Rinne, U./Zimmermann, K. F.: Anonymisierte Bewerbungsverfahren, in: IZA Research Report Nr. 27/2010

Krell, G./Riedmüller, B./Sieben, B./Vinz, D.

(Hg.): Diversity Studies. Frankfurt am Main

2007.

Krell, G./Sieben, B.: Diversity Management, in: Massing, P. (Hg.): Gender und Diversity. Schwalbach/Ts. 2010.

Loden, M:/Rosener, J. B.: Workforce America. Managing Employee Diversity as a vital Resource. Homewood 1991
Perko, G:/Czollek, L. C.: Gender und Diversity gerechte Didaktik: ein intersektionaler Ansatz, in: Magazin erwachsenenbildung.at, 3/2008. Stock-Homburg, R.: Personalmanagement. 2. Auflage Wiesbaden 2010.

Thomas, R. R. Jr.: Redefining Diversity. New York 1996.

Vedder, G.: Die historische Entwicklung von Managing Diversity in den USA und in Deutschland, in: Krell, G./Wächter, H. (Hg.): Diversity Management. Impulse aus der Personalforschung. München 2006.

\section{Die Zukunft des Sozialen - in Europa? \\ Soziale Dienste und die europäische Herausforderung Herausgegeben von RAin Dr. Ulla Engler und Dr. Joachim Rock 2012, ca. 100 S., brosch., ca. 19,- $€$ ISBN 978-3-8329-5689-9 \\ (Edition Sozialwirtschaft, $B d$. 30) Erscheint ca. Oktober 2012}

\section{Nomos}

